GaAs nanowires and related prismatic heterostructures

This article has been downloaded from IOPscience. Please scroll down to see the full text article.

2009 Semicond. Sci. Technol. 24113001

(http://iopscience.iop.org/0268-1242/24/11/113001)

View the table of contents for this issue, or go to the journal homepage for more

Download details:

IP Address: 128.178.106.14

The article was downloaded on 28/04/2010 at 07:23

Please note that terms and conditions apply. 


\title{
TUTORIAL
}

\section{GaAs nanowires and related prismatic heterostructures}

\author{
D Spirkoska $^{1}$, G Abstreiter ${ }^{1}$ and A Fontcuberta i Morral ${ }^{1,2}$ \\ ${ }^{1}$ Walter Schottky Institute, Technische Universität München, Am Coulombwall 3, 85748 Garching, \\ Germany \\ ${ }^{2}$ Laboratoire des matériaux Semiconducteurs, Instiut des Matériaux, Ecole Polytechnique Fédérale de \\ Lausanne, 1015 Lausanne, Switzerland \\ E-mail: spirkoska@wsi.tum.de
}

Received 2 June 2009, in final form 3 September 2009

Published 9 October 2009

Online at stacks.iop.org/SST/24/113001

\begin{abstract}
The growth of GaAs nanowires by the gallium-assisted method with molecular beam epitaxy (MBE) is presented in this review article. The structure of the grown nanowires was investigated by means of scanning and transmission electron microscopy as well as Raman spectroscopy. Their optical properties were revealed by performing photoluminescence measurements at the single nanowire level. Furthermore, by tuning the MBE conditions to planar growth, quantum heterostructures on the side facets of the nanowires were achieved. High-resolution transmission electron microscopy proved that the grown heterostructures have epitaxial precision, while photoluminescence measurements showed that they possess excellent optical quality. These quantum heterostructures constitute templates for developing novel nanowire based devices, such as a high electron mobility one-dimensional transistor or third generation solar cells.
\end{abstract}

(Some figures in this article are in colour only in the electronic version)

\section{Introduction}

Semiconductor nanowires are interesting nanoscale objects. Their study constitutes a research field that has expanded very fast in the last decade. Semiconductor nanowires offer numerous possibilities for studying a variety of physical phenomena arising from their peculiar geometry: carrier confinement, variations in the band structure, coherence of charge carriers, crystal phase changes due in part to the large surface to volume ratio. It is believed that they will play an important role in scaling down the dimensions of future optoelectronic devices, such as lasers and light emitting diodes. Thanks to their high surface to volume ratio, they are excellent systems for building the future generation of biological and chemical sensors. Nanoscale lasers, light emitting diodes and sensors have been demonstrated in the past which proves that the added functionality of semiconductor nanowires is a real fact and not just a dream [1-7].
A further interesting aspect of nanowires is that they enable the integration of lattice-mismatched materials, which has been an experimental issue in the last decades [8,9]. Due to the small diameter the strain can be released efficiently, without the formation of dislocations and/or stacking faults, which is the case in thin films. This point has already been theoretically considered by several authors [10-13]. This freedom in the design of nanowire heterostructures further increases their functionality and possible fields of applications.

Nanowires are regularly grown by using the vapor-liquidsolid (VLS) growth method. The method was applied for the first time more than four decades ago by Wagner and Ellis [14]. Since then, it is the most used method for the fabrication of whiskers and nanowires. The central point of this method is the use of a liquid metal droplet acting as a catalyst where preferential decomposition of growth precursors in the gas phase occurs. As soon as the droplet becomes supersaturated the material starts to precipitate at the interface between the liquid droplet and the solid substrate. The continuous 
precipitation of material through the droplet results in the continuous longitudinal growth of the nanowire. The metal droplet stays in all cases at the tip of the nanowire. Gold is the widely used metal as a catalyst droplet, and it works very well with a wide range of semiconductor materials: oxides, groups IV and III-V semiconductors. On the other hand, $\mathrm{Au}$ is generally an unwanted impurity because of its large diffusivity and because it produces deep level traps in the band gap of the semiconductor [15]. This can significantly harm the opto-electronic properties of the devices. Therefore, growth of nanowires catalyst-free or by using alternative catalysts has been extensively studied in the past years [1619]. Recently, growth without an external catalyst has been demonstrated both by using metal organic chemical vapor deposition (MOCVD) and molecular beam epitaxy (MBE) [20-23].

From these two techniques MBE is particularly interesting since it offers the possibility for growth of high purity materials with excellent crystal quality [24-26]. Furthermore, one can combine the nanowire growth with the planar layer-by-layer kind of growth, which leads to the development of more complex heterostructures on the nanowire facets. These kinds of radial heterostructures have been obtained previously from different groups by applying different growth methods [22, 27].

In this work, the growth of nanowires with MBE by the so-called Ga assisted VLS growth method is presented. The obtained nanowires exhibit excellent optical quality, as will be shown. Epitaxy directly on the side facets of the nanowires, resulting in a formation of so-called prismatic quantum heterostructures will also be shown. These add further functionality to the nanowires.

The paper is structured in the following manner: the first part is devoted to GaAs nanowires. Details of the growth method are presented, followed by the results from the structural characterization of the nanowires. Two main characterization tools are considered: transmission electron microscopy (TEM) and Raman spectroscopy. The optical properties of the grown GaAs nanowires are also discussed. The second part deals with the epitaxy on the side walls of the nanowires, leading to formation of prismatic quantum heterostructures. High-resolution TEM micrographs are presented to prove the precision of this nanoepitaxy. The optical properties are discussed at the end. The paper is concluded in the third section, where some future applications of the bare GaAs nanowires, as well as the prismatic heterostructures are discussed.

\section{GaAs nanowires}

\subsection{Growth principles of GaAs nanowires}

In order to find the conditions leading to catalyst-free galliumassisted growth of nanowires by MBE, the phase diagram of the Ga-As system has to be considered. This enables us to deduce the conditions (temperature, As pressure) under which a liquid $\mathrm{Ga}$ droplet will exist in equilibrium with a stoichiometric GaAs surface. The $p-x$ projection of the phase

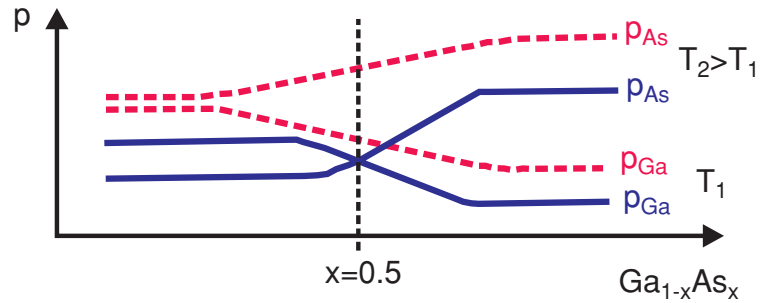

Figure 1. Schematic of the $p-x$ projection of the phase diagram of the $\mathrm{Ga}-\mathrm{As}$ system. For $\mathrm{T}_{2}$ higher than $\mathrm{T}_{1}$ the partial pressure of $\mathrm{As}$ increases faster than the partial pressure of $\mathrm{Ga}$, leading to evaporation of As and formation of Ga rich surface. Reproduced from [28].

diagram of the Ga-As system is shown in figure 1[28]. Here, $x$ corresponds to the composition of the compound, being $x=0.5$ the stoichiometric for GaAs.

At low temperatures the vapor pressures of As and Ga coincide for stoichiometric GaAs $(x=0.5)$. For compositions below and above $x=0.5$, the vapor pressures do not coincide in a way that $\mathrm{Ga}$ or As are selectively evaporated until stoichiometry is reached. At high temperatures, the vapor pressure of As increases with respect to Ga, leading to preferential evaporation of As in the surface. In these conditions, the surface becomes $\mathrm{Ga}$ rich and $\mathrm{Ga}$ droplets are formed. The transition temperature from the low to the high temperature regime is called the congruent temperature. The Ga droplets can remind of the gold droplets used in the VLS method for the nucleation and growth of the nanowires. Indeed, the droplets are in equilibrium with the GaAs surface and serve as a sink for precipitation of As atoms, which are alloyed with Ga, thereby forming stoichiometric GaAs [29]. This means that temperatures higher than the congruent and low As pressures may be essential point for nucleation of nanowires.

The growth was realized in a Gen II MBE system using $2^{\prime \prime}$ GaAs wafers as substrates. Prior to growth the substrates were heated up to $650{ }^{\circ} \mathrm{C}$ to desorb any remnant molecules from the surface. Afterward the temperature was lowered to the growth temperature of $630{ }^{\circ} \mathrm{C}$. An $\mathrm{As}_{4}$ beam equivalent pressure (BEP) of $3.5 \times 10^{-6}$ mbar and a Ga deposition rate of $0.025 \mathrm{~nm} \mathrm{~s}^{-1}$ were used.

As discussed here above, the temperature and arsenic beam flux conditions leading to the formation of gallium droplets are known. However, for the formation of nanowires the wetting properties need to be considered too. Indeed, if bare GaAs is used as a substrate, the droplets will spread on the GaAs surface and the growth will not proceed outward of the substrate surface. This growth method is based on the well-studied droplet epitaxy growth technique for formation of island of III-V materials as well as ring structures [3033]. In our experiments, formation of a mesocoral structure is observed, where besides ring formation a vertical growth in the center with a height of only several tens of nanometers also occurs as shown in figure $2(a)$. This can be overcome by using a gallium non-wetting substrate that at the same time enhances the diffusion of the $\mathrm{Ga}$ ad-atoms. Previous experiments of deposition of $\mathrm{GaAs}$ on $\mathrm{SiO}_{2}$-coated GaAs substrates showed 


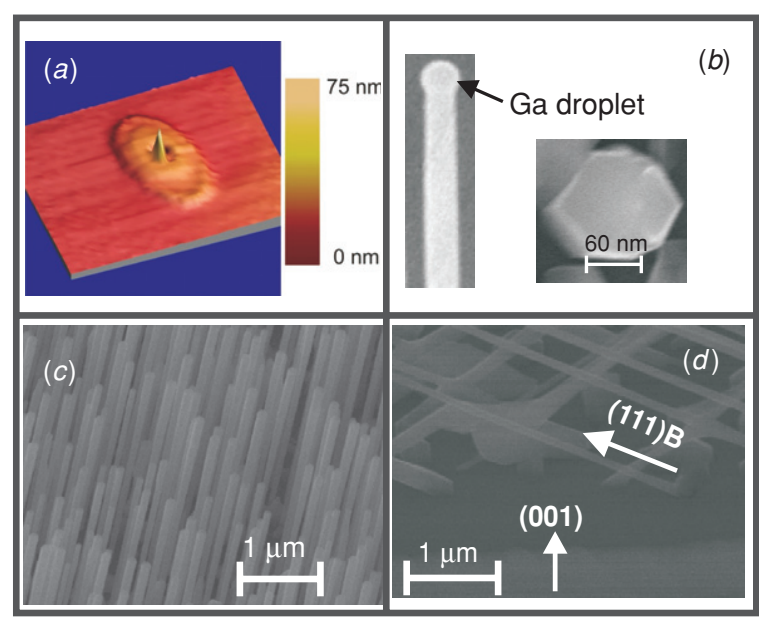

Figure 2. (a) Mesocoral structure formed when bare GaAs substrate is used for the growth. (b) (Left) Single GaAs nanowire with a Ga droplet at the tip. (Bottom) SEM micrograph from a broken nanowire revealing its hexagonal geometry. (c) GaAs nanowires grown on (1 11 1)B substrate. The wires grow vertically with respect to the substrate. $(d) \mathrm{GaAs}$ nanowires grown on $\left(\begin{array}{lll}0 & 0 & 1\end{array}\right)$ substrate. The growth axes of the wires have an angle of $35^{\circ}$ with respect to the substrate.

that for temperatures above the congruent point the sticking coefficient of $\mathrm{Ga}$ on the oxide is negligible and the diffusion length is several micrometers long [34]. For this reason, we have used GaAs substrates covered with approximately $35 \mathrm{~nm}$ of sputtered $\mathrm{SiO}_{2}$. The cleanliness of the substrates is a crucial issue. Prior to growth the substrates are cleaned by a short etching in buffered oxide, blown dry with nitrogen and immediately transferred into the growth chamber. Covering the $\mathrm{GaAs}$ substrate with $\mathrm{SiO}_{2}$ resulted in growth of nanowires as can be seen in figures $2(c)$ and $(d)$. On the tip of every nanowire a droplet is observed as shown in figure 2(b) (left). In the bottom of figure $2(b)$ the shown SEM micrograph of a broken nanowires reveals its hexagonal geometry. The micrographs shown in figures $2(c)$ and $(d)$ are recorded on two different samples with the only difference being the orientation of the GaAs substrate. The substrate orientation

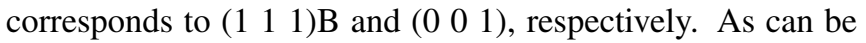
seen on the micrographs, this resulted in growth of nanowires standing perpendicular on the substrate in the first case, and in nanowires taking an angle of $35^{\circ}$ with the substrate in the second case. This proves the existence of relation between the nanowire growth direction and the crystalline structure of the substrate beneath the $\mathrm{SiO}_{2}$ layer. We believe and have some experimental evidence that the interaction of reactive Ga with the $\mathrm{SiO}_{2}$ pin holes creates nanocraters which are prerequisite for nucleation of nanowires [35].

With the purpose of getting more insight into the growth mechanisms, we have explored in more details the parameter space of growth conditions. This includes change of the As pressure as well as the $\mathrm{Ga}$ deposition rate while keeping the temperature always constant. We have changed the $\mathrm{As}_{4} \mathrm{BEP}$ in a pressure range from $3 \times 10^{-7}$ mbar up to $3.5 \times 10^{-6}$ mbar. For $\mathrm{As}_{4}$ pressures higher than $3.5 \times 10^{-6}$ mbar the Ga droplets are crystallized and multifaceted GaAs crystals are obtained instead. In figure 3(a), the dependence of the growth rate from the $\mathrm{As}_{4}$ BEP for a Ga deposition rate of $0.025 \mathrm{~nm} \mathrm{~s}^{-1}$ is shown. We can clearly see that the growth rate increases linearly with the $\mathrm{As}_{4}$ BEP. This leads to the conclusion that the arsenic governs the growth [36]. The obtained results are plotted only until pressures of $8 \times 10^{-7} \mathrm{mbar}$, since for higher pressures the dispersion of the length is significantly increased which causes difficulties in determining the average length of nanowires. In figure $3(b)$, the dependence of the length of the nanowires from the growth time for different $\mathrm{Ga}$ deposition rates is plotted. The length increases linearly with time and it is not influenced by the $\mathrm{Ga}$ deposition rate. This further proves that the role of the $\mathrm{Ga}$ is to act as a liquid reservoir for precipitation of As atoms, but it is the As that governs the growth. For very high Ga deposition rates, such as $0.081 \mathrm{~nm} \mathrm{~s}^{-1}$ the nanowires exhibit inverse tapered geometry as shown with the scanning electron micrograph in the inset of figure $3(b)$. This is a consequence of the fact that with the time the droplet increases its size as a result of the large gallium arrival rate, which essentially leads to the increase of the diameter during the growth and therefore to an inverted tapering geometry.

The growth method is schematically shown in figures 4(a) and $(b)$. The enhanced diffusion length of the $\mathrm{Ga}$ atoms on the $\mathrm{SiO}_{2}$ surface as well as the non-wetting properties lead to formation of $\mathrm{Ga}$ droplets. On this high temperature the $\mathrm{As}_{4}$ molecules impinging on the oxide surface are desorbed back in the vacuum, while the one impinging of the liquid Ga droplets decompose into As atoms which diffuse through the droplet following the As gradient where they alloy with $\mathrm{Ga}$ atoms and form GaAs. The Ga droplets are constantly enriched by other Ga atoms that are diffusing through the oxide and along the nanowire walls.

\subsection{Structural characterization}

Structural characterization of the grown nanowires was performed by two methods: transmission electron microscopy (TEM) and Raman spectroscopy.

2.2.1. TEM characterization. The structure of the grown wires was first investigated by TEM. This is a powerful tool for material characterization, which can give a valuable information about the growth direction, the crystal orientation of the side facets as well as the structural quality of the material.

TEM characterization requires suitable sample preparation. It is important that a majority of electrons are transmitted through the sample without major loss of energy. For crystalline samples, this is achieved if the thickness is not thicker than about $100 \mathrm{~nm}$. Additionally, it is required that the whole sample lies on the same plane, so that the microscope can focus equally through the whole sample. For this reason, the nanowires were removed from the original substrate by mechanically breaking them off with a sharp blade and mixing them with a volatile liquid such as hexane. A drop of solution was deposited on a copper TEM grid with a holey carbon membrane, which after evaporation resulted in nanowires lying on the grid. Only nanowires lying on the holes can be measured. A schematic representation of a solution of nanowires deposited on the grid is shown in figure 5 . 

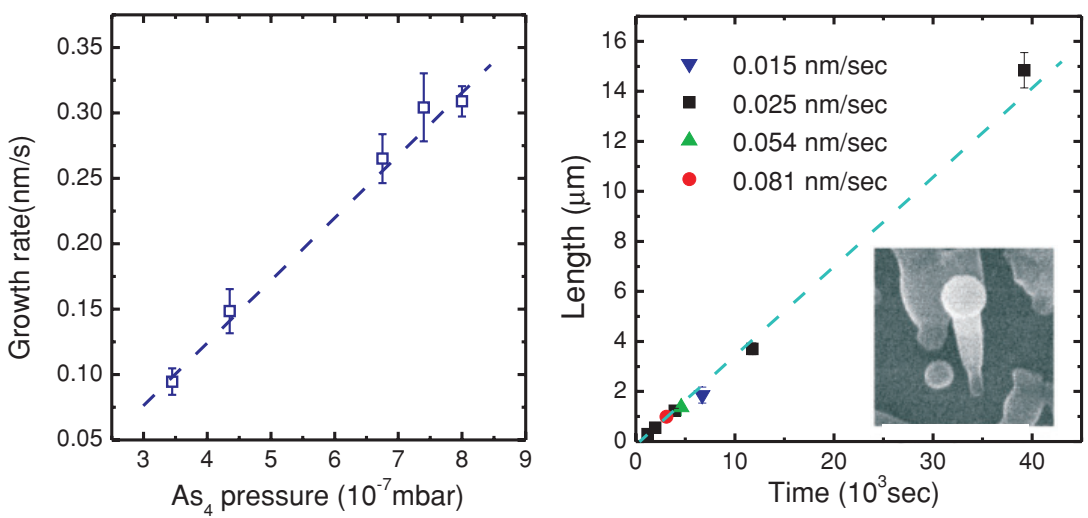

Figure 3. (a) Dependence of the growth rate from the As BEP. The points represent the experimental data, while the dashed curve is a linear fit. A clear linear dependence is observed. $(b)$ Time dependence of the length of the nanowires for different Ga deposition rates. The inset shows a tapered wire. The tapering occurs only for high Ga deposition rates.

$(a)$

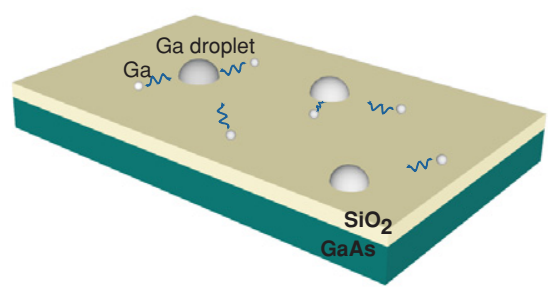

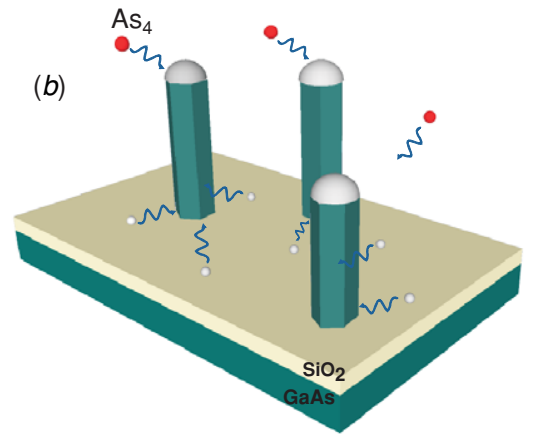

Figure 4. Schematic of the growth process: (a) Ga atoms are able to diffuse on the $\mathrm{SiO}_{2}$ surface and collect into droplets. For better clarity of the schematic figure the above discussed pin holes formed in the $\mathrm{SiO}_{2}$ layers are not drawn. $(b)$ These droplets act as sinks, where As atoms coming from the vapor precipitate and diffuse toward the solid-liquid interface. As a consequence a nanowire growth occurs. The Ga droplet at the tip is constantly enriched with $\mathrm{Ga}$ atoms diffusing at the $\mathrm{SiO}_{2}$ surface and the wall of the nanowire.

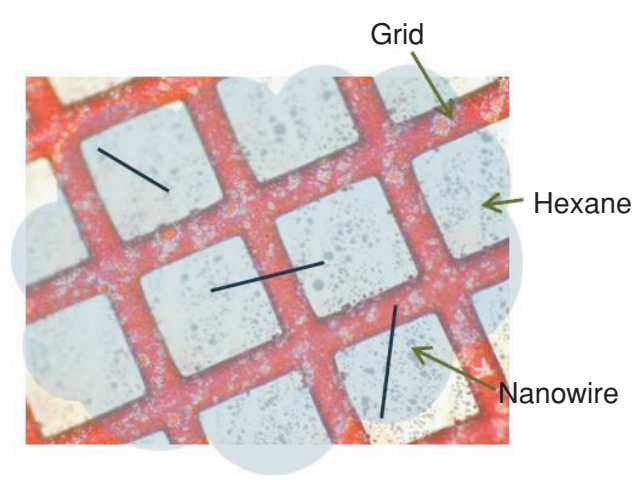

Figure 5. Schematic representation of a sample prepared for TEM characterization. Nanowire solution prepared in hexane is deposited on a TEM grid with a holey carbon membrane.

In figure 6(a), a typical bright field (BF) TEM micrograph of a single nanowire is presented. For a BF imaging mode an aperture is placed in the back focal plane of the objective lens. This allows only direct electrons to pass which create the image. In contrast, when the direct electrons are blocked with an aperture, while the diffracted are allowed to pass, the corresponding TEM image is dark field image [37]. As can be seen the nanowire has several dark stripes perpendicular to the growth direction, with different spacing. A high- resolution TEM (HRTEM) was further performed to determine the growth direction. It was found that the nanowires have the zinc-blende structure and grow in the $(1-11)$ growth direction. To characterize the dark and the bright stripes we further did a HRTEM on these regions. A typical HRTEM micrograph is presented in figure $6(b)$ where the inset shows a magnified view of the selected region. The Fourier transform (power spectrum) of the HRTEM micrograph was calculated numerically on both sides of the contrast stripe. The results are presented in figure $6(c)$. As can be seen the two sides of the interface correspond to growth along the $(1-11)$ growth direction. On each side of the interface the adjacent facets correspond to [ [ $\left.\begin{array}{lll}1 & 1 & 0\end{array}\right]$ and $\left[\begin{array}{lll}0 & 1 & 1\end{array}\right]$ surfaces. These kind of defects are referred to as a twin defect which corresponds to a $60^{\circ}$ rotation of the zinc-blende structure along the growth axis. Twin defects are commonly found in the structure of III-V nanowires, and it has already been shown that they have a tremendous effect on their optical properties [38].

\subsubsection{Raman spectroscopy characterization. Raman} spectroscopy as a non destructive characterization tool is extensively applied for characterization of low-dimensional systems, such as nanowires and nanocrystals. It provides valuable information about their structural quality [41, 42]. Nanowires are particularly interesting systems for Raman 


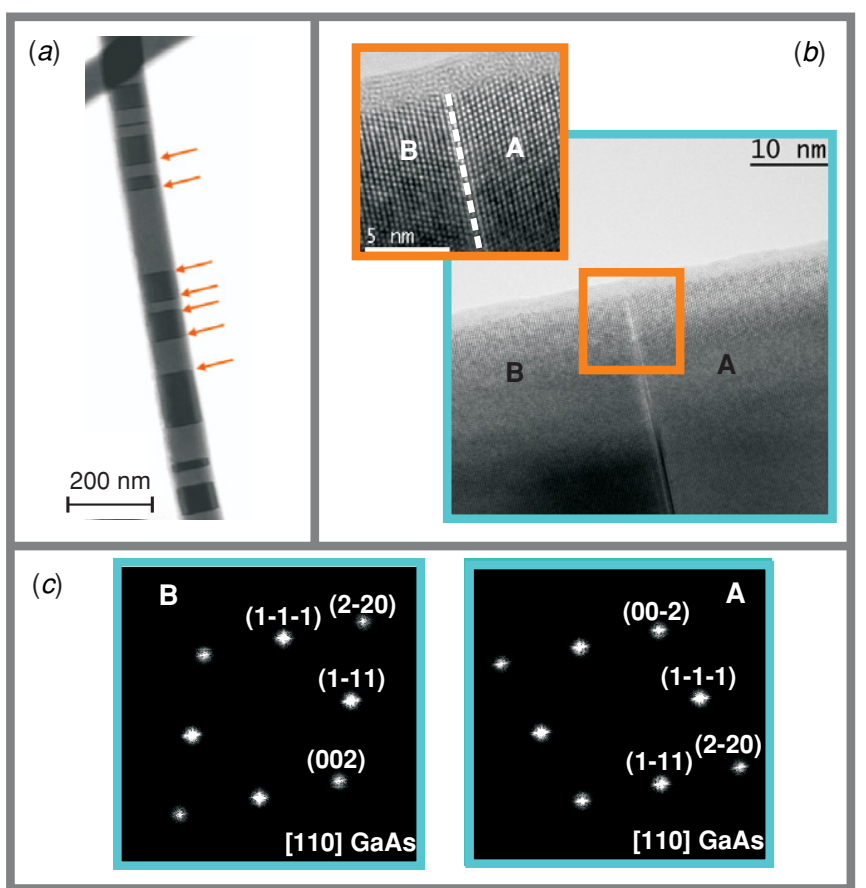

Figure 6. (a) Bright field TEM micrograph of a single GaAs nanowire. Dark stripes perpendicular to the growth direction are clearly observable. (b) High-resolution TEM at the interface of the contrast stripe. The inset shows a magnified view of the selected region. (c) Fourier transform (power spectrum) of the high-resolution TEM micrograph.

spectroscopy characterization due to the large surface-tovolume ratio. As we have shown previously, Raman spectra from GaAs nanowires show additional features which are not observed in bulk GaAs [39].

For the measurements the nanowires were removed from the original substrate and transferred to a Si substrate. This was done by applying a mechanical friction between the two substrates which resulted in a partial orientation of the nanowires. Depending on the strength of the friction, the density of the transferred nanowires can be varied, e.g. for a stronger friction, a higher density is obtained. This can be seen in figure 7(a) where a SEM micrograph of the transferred wires is shown. The measurements were performed at room temperature using the $488 \mathrm{~nm}$ line from an $\mathrm{Ar}+$ laser. A microscope objective $(50 \times)$ focused the laser on the sample in a spot of several micrometers in a size. We estimate that only 10-20 wires can be focused in the laser spot. The same objective lens collected the scattered light, which with the help of mirrors was guided into the entrance slit of a spectrometer and further analyzed with a Si CCD. In Raman experiments in general, the intensity of the scattered light is very weak as well as the frequency difference between the elastically and Raman scattered light. It is for this reason that the use of a triple spectrometer is very important, in order to filter in an effective way the reference line of the incident laser. For the measurements, a DILORXY spectrometer was used with a spectral resolution of (1.5-2) $\mathrm{cm}^{-1}$. A schematic drawing of the experimental setup is shown in figure $7(c)$. The scattering geometry is shown in figure $7(b)$. The wires are probed

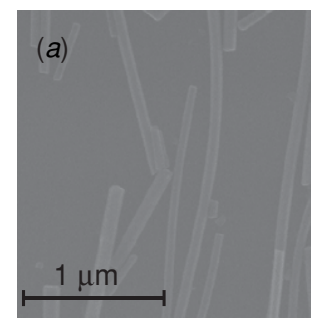

(c)

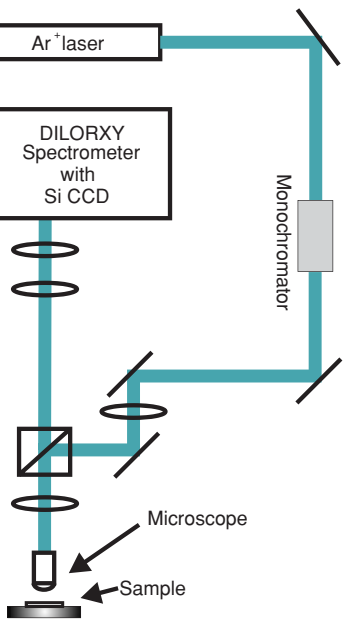

Figure 7. (a) Scanning electron micrograph from GaAs nanowires transferred on Si substrate. Due to the low density only 10-20 nanowires are probed during the measurement. $(b)$ Schematic of the scattering geometry. The side facets of the nanowire belong to the $\left\{\begin{array}{lll}1 & 1 & 0\end{array}\right\}$ crystal family. (c) Schematic of the Raman experimental setup.

in the backscattering geometry. From TEM measurements we know that the side facets belong to the $\left\{\begin{array}{lll}1 & 1 & 0\end{array}\right\}$ crystal family. In the backscattering geometry the longitudinal optical phonon (LO) is forbidden according to Raman selection rules, while the transversal optical phonon (TO) is allowed [40]. Since the top facets contribute most to the signal, the spectra should present rather strong TO signal and much weaker LO signal.

Typical Raman spectrum from nanowires with average diameter of $89 \mathrm{~nm}$ is shown in figure 8(a) upper panel. For a comparison on the lower panel the Raman spectrum from (1 1 1)B GaAs is also plotted. On the Raman spectrum from the nanowires two peaks position at $268 \mathrm{~cm}^{-1}$ and $292 \mathrm{~cm}^{-1}$ are clearly observable. According to the reference GaAs sample these peaks are identified as the TO and LO phonon, respectively. They have a full width at half-maximum (FWHM) of approximately $4 \mathrm{~cm}^{-1}$ and their position corresponds very well to the position of the TO and LO in bulk GaAs. This leads to the conclusion that the nanowires exhibit good structural quality and are free from stress and defects.

A third peak is also observable at the low frequency side from the LO phonon. A detailed analysis on this peak which will be presented in the following allowed its attribution as scattering from surface optical phonon (SO). Mathematical expression for the dispersion relation in the case of an infinitely long cylinder is given with [43-45].

$$
\omega_{S O}^{2}=\omega_{T O}^{2}+\frac{\omega_{p}^{2}}{\epsilon_{\infty}-\epsilon_{m} f(q r)},
$$

where

$$
f(q r)=\left(I_{0}(q r) K_{1}(q r)\right) /\left(I_{1}(q r) K_{0}(q r)\right),
$$

with $\omega_{p}$ being the screened ion plasma frequency given by

$$
\omega_{L O}^{2}=\omega_{T O}^{2}+\omega_{p}^{2} / \epsilon_{\infty}
$$



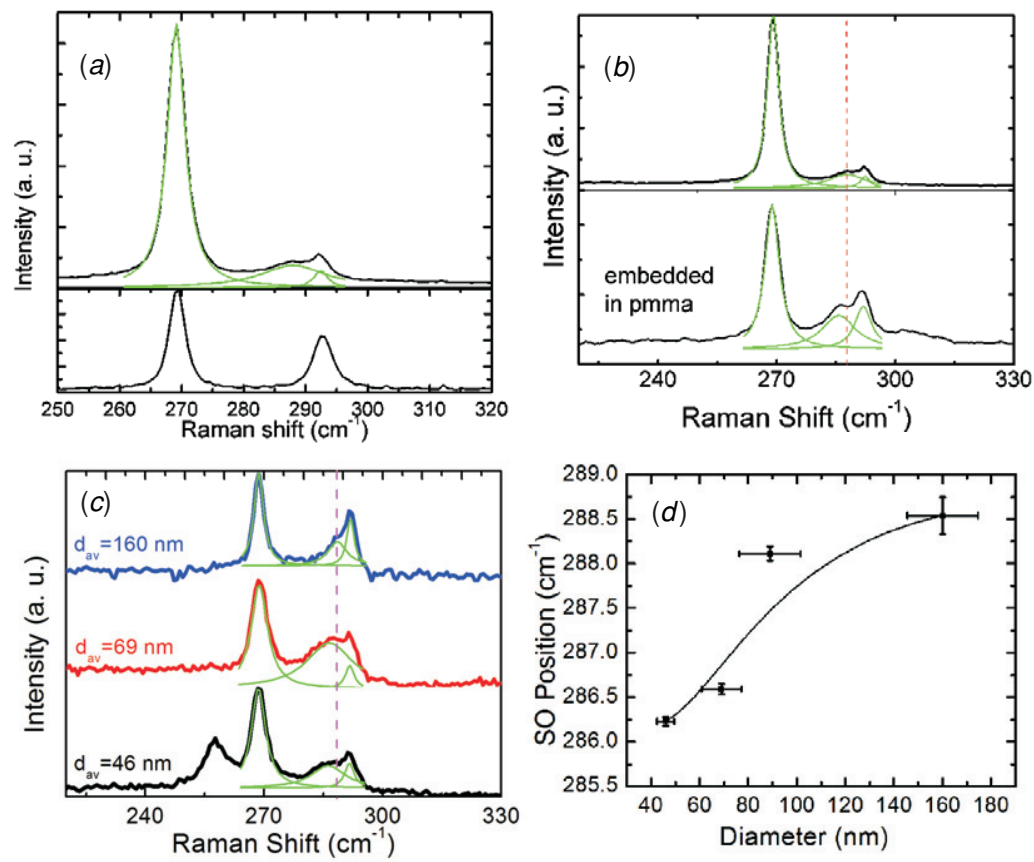

Figure 8. (a) The Raman spectrum from GaAs nanowires are shown on the upper panel, while the Raman spectrum from a reference bulk (1 111 ) GaAs substrate is shown on the lower panel. The spectrum is fitted using a multiple Lorentzian fit. (b) Raman spectrum from nanowires in air is plotted on the upper panel. On the lower panel the Raman spectrum from nanowires embedded in PMMA is shown. The (red) dashed line is a guide to the eye. Downshift is clearly visible. (c) Representative Raman spectra from nanowires with different diameters. $(d)$ Dependence of the SO position from the diameter. The black line corresponds to theoretical calculations according to [43].

where $I_{j}(q r), K_{j}(q r)$ are the modified Bessel functions, $q$ is the phonon wave vector, $\epsilon_{\infty}$ is the high-frequency dielectric constant of the material and $\epsilon_{m}$ is the dielectric constant of the surrounding material. The frequency of the SO phonon depends on the dielectric constant of the material surrounding the nanowires as well as on the diameter of the nanowires. In order to investigate the dependence on the dielectric constant the nanowires were covered with a layer of PMMA (polymethyl metacrylat) that has a dielectric constant of 2.8. In figure $8(b)$, in the upper panel we have plotted again the Raman spectrum from the nanowires in the air, while in the lower panel we have plotted the corresponding spectrum in a PMMA environment. The red dashed line is a guide to the eye. Indeed covering the nanowires by a layer of PMMA caused down sift of the position of the SO phonon of $1.8 \mathrm{~cm}^{-1}$. In order to test whether the change in the diameter of the nanowires will also cause a shift in the position of the SO phonon, samples with nanowire diameters ranging from $50 \mathrm{~nm}$ up to $160 \mathrm{~nm}$ were grown. This was done simply by varying the growth time, but keeping all other growth conditions the same. Each sample was characterized with diameter distribution between $10 \%$ and $20 \%$. Representative Raman spectra from samples with average diameters of $160 \mathrm{~nm}, 69 \mathrm{~nm}$ and $49 \mathrm{~nm}$ are shown in figure $8(c)$. As is shown the position of the TO and the LO phonon does not depend from the diameter, while the SO phonon downshifts. Furthermore in the sample with the thickest diameter the SO phonon appears only as a small shoulder, but on the samples with smaller diameter, meaning larger surface-to-volume ratio, is clearly pronounced.

In the sample with the smallest diameter another peak appears on the left side from the TO phonon. This could come due to the presence of the twin defects as seen from the TEM measurements. Since the wires with small diameter also have smaller length the twin defect consist significant percent from the nanowire length. Further investigation in this direction is currently ongoing.

In figure $8(d)$, the dependence of the SO phonon from the diameter is plotted. The black line corresponds to theoretical calculations considering cylindrical nanowires according to [43]. The shift in the position of the SO phonon is not very strong, but this can also be attributed to the diameter distribution in the samples. According to the theoretical calculations there should be a larger shift, especially for the nanowires with larger diameters. This can also depend from the cross section of the nanowires: the wires in this case have hexagonal cross section, while the theoretical calculations are performed for cylindrical nanowires. Recently, it was shown that the cross section of the nanowires also plays a significant role when calculating the dispersion relation of the SO phonon [46].

\subsection{Optical properties}

The optical properties of single nanowires were assessed by means of photoluminescence spectroscopy (PL). For the measurements a confocal microscope experimental setup was used $^{3}$. A schematic drawing of the experimental setup is shown in figure 9.

The $633 \mathrm{~nm}$ line of a HeNe laser was used as an excitation laser. The laser light is coupled into a single-mode optical fiber

3 Commercially available Confocal Microscope from Attocube is used for performing photoluminescence measurements. 


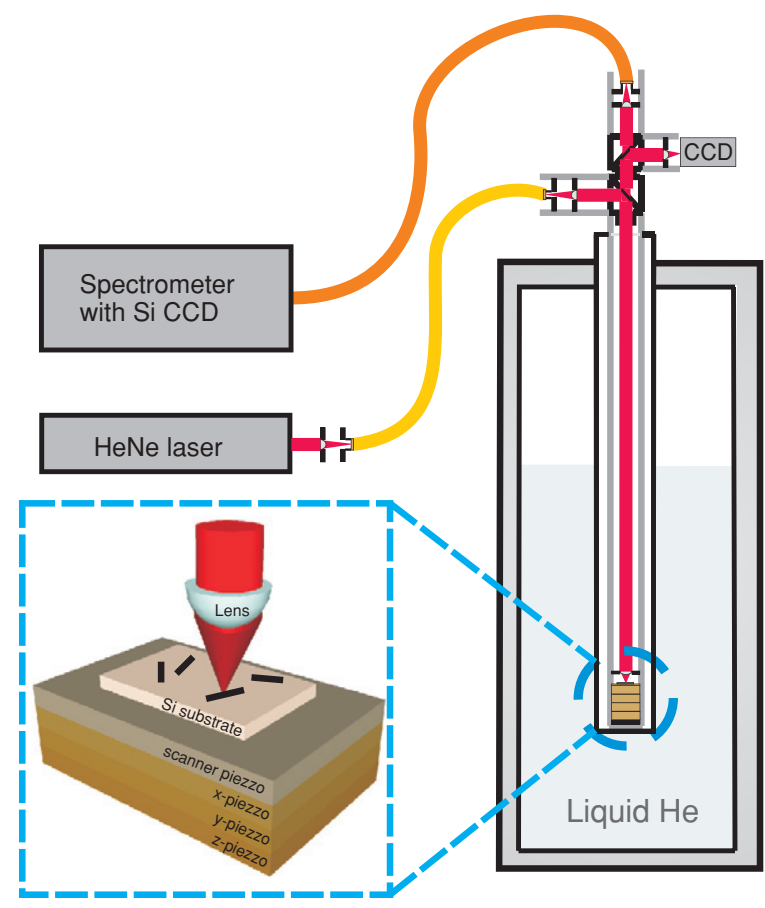

Figure 9. Schematics of the confocal microscope setup used for photoluminescence measurements. The inset in the lower part shows the sample lying on top of the piezzo positioners and the laser light focused with the confocal lens.

that guides the light to the top of the microscope, where the light is decoupled from the fiber and with the help of a lens a parallel beam of light is produced. The parallel beam falls onto a beam splitter (splitting ratio of $50 \%$ ) and one part of it is directed toward the lower part of the microscope. The lower part of the microscope is immersed in liquid He, with a temperature of $4.2 \mathrm{~K}$. There with the help of a lens (numerical aperture of 0.65) the laser is focused on the sample as a spot with a diameter of less than $1 \mu \mathrm{m}$. The same lens collects the reflected laser light as well as the emitted photoluminescence from the sample which travels toward the upper part of the microscope. After going through two beam splitters the signal is coupled into a multi-mode detection fiber. The core of the fiber has the role of a blocking pin hole, rejecting the out of focus light. The light that is coupled out from this fiber is focused either on a Si photodiode for measuring the intensity of the reflected light or on the entrance slit of a spectrometer equipped with Si CCD for spectral analysis. The sample is mounted on a top of a four piezzo positioners as seen in the inset of the bottom of figure 9. Three of the positioners provide movement in the $z-, x$ - and $y$-directions, while the top positioner can move in the $x-y$-plane with a range of $30 \times 30 \mu \mathrm{m}$.

For the measurements the nanowires were removed from the growth substrate and transferred on small $\mathrm{Si}$ pieces. As previously presented in the section dedicated to Raman spectroscopy, this was done by applying small mechanical contact between the substrates and resulted in single nanowires lying separately from each other on the Si piece. Optical microscope image from the sample prepared for measurements is shown in figure $10(a)$. In order to isolate single nanowire
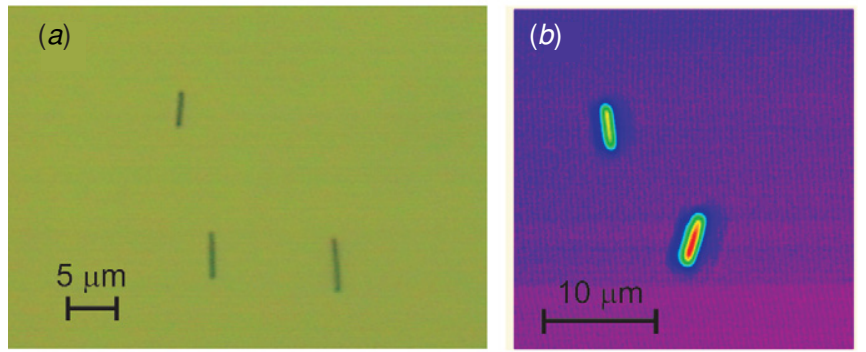

Figure 10. (a) Image of the sample with transferred nanowires taken with optical microscope. (b) Scanning reflection image of the sample. Single nanowires can be clearly observable.

first scanning reflectivity measurements were realized. For this, the sample is scanned below the fixed laser spot in a field with dimensions of $30 \times 30 \mu \mathrm{m}$. The intensity change of the reflected light from the sample is detected with the $\mathrm{Si}$ photo diode and the surface of the sample is reconstructed as shown in figure $10(b)$ where single nanowires can be clearly distinguished.

After isolating single nanowire the laser spot is focused on it and the emitted PL is detected and analyzed with the Si CCD equipped spectrometer. A typical PL spectra from a single nanowire are shown in figure 11 $(a)$. As can be seen the spectra have one single emission line centered at $1.51 \mathrm{eV}$ with a full width at half-maximum of $6 \mathrm{meV}$ which corresponds well to the free exciton emission from bulk undoped GaAs. It should be stressed that these data are exceptional among the nanowires and it should also be taken into the account that the surface of the nanowires was not passivated and surface states might be present. The nanowires were also characterized with spatially resolved PL, when the laser spot was moved along the nanowire in steps of $250 \mathrm{~nm}$ and each time the emission was recorded and analyzed. A typical spatially resolved scan is shown in figure $11(b)$ where the emitted light with respect to the length of the nanowire is plotted. It can be clearly seen that the nanowire emits along its entire length which further corroborates the use of MBE for growth of high-purity nanowires [47].

\section{Prismatic heterostructures}

The MBE technique offers versatility of growth modes from one to two dimensional. This can be utilized to add complexity to the nanowire structures. As we have shown recently with carefully engineering of the growth conditions the side facets of the nanowires can be overgrown with successive layers of GaAs and AlGaAs [48, 49]. In this case, when a thin GaAs layer is sandwiched between two AlGaAs layer prismatic quantum wells ( $\mathrm{p}-\mathrm{QWs}$ ) will be formed. As we will show this adds further functionality of the nanowires and these structures can present a basis for formation of more complex quantum wire-quantum dot-based heterostructures.

\subsection{Growth of prismatic heterostructures}

As was shown previously, the grown nanowires have hexagonal geometry and their side facets belong to the 

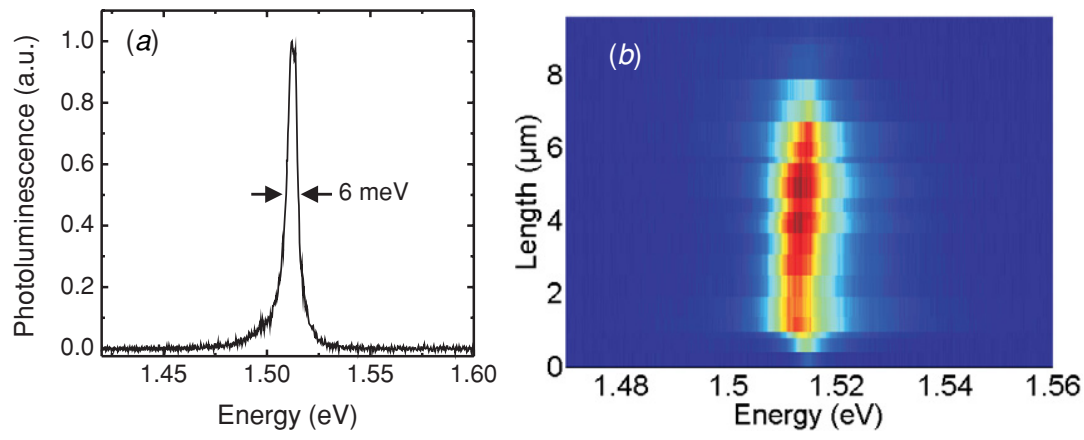

Figure 11. (a) Typical PL spectra from single GaAs nanowire recorded at $4.2 \mathrm{~K}$. The full width at half-maximum is $6 \mathrm{meV}$. (b) Spatially resolved PL emission from the nanowire. PL emission from the entire length of the nanowire was observed.

(a)

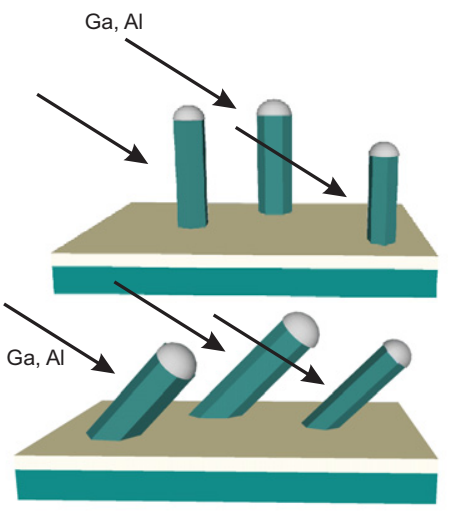

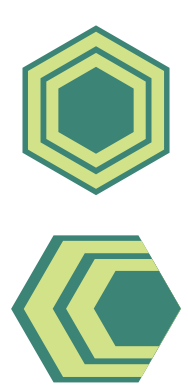

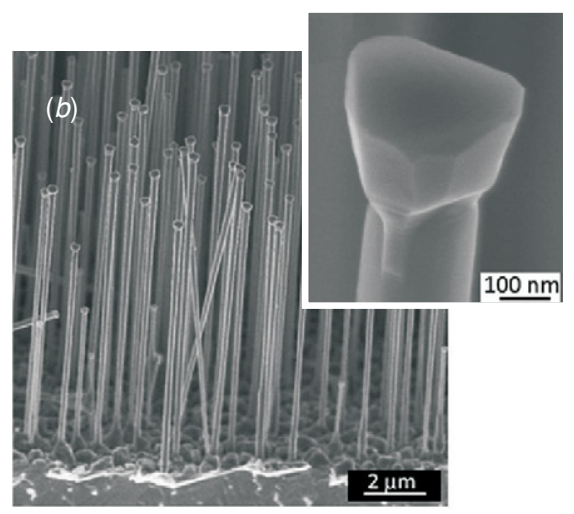

Figure 12. (a) Schematic of the growth process of the p-QWs. For perpendicular nanowires six quantum wells with equal thickness should be formed, while for angled nanowires quantum wells with different thicknesses will form. SEM micrographs from overgrown GaAs nanowires. In the inset a magnified view of a single nanowire is shown. As can be seen the Ga droplets are fully crystallized.

$\left\{\begin{array}{lll}1 & 1 & 0\end{array}\right\}$ crystal family. This means that the growth of the p-QWs is done on the $\left\{\begin{array}{lll}1 & 1 & 0\end{array}\right\}$ surfaces which are non-polar. Previous research on (1 110$)$-oriented GaAs substrates has shown that it is more difficult to obtain smooth morphology on these surfaces due to the lack of surface reconstruction [50]. The nanowires were overgrown immediately after they reached a certain length by changing the growth conditions corresponding to planar MBE growth mode. This required the As BEP to be increased up to $4 \times 10^{-5}$ mbar.

The overgrowth was done both on nanowires that stand perpendicular on the growth substrate and nanowires that are angled corresponding to the substrate. Due to the directionality of the molecular beams, deposition occurs only on the exposed surfaces. In the case of the perpendicular nanowires and additional rotation of the substrate during growth, each side faces will receive the same material flux resulting in a formation of six quantum wells having the same thickness arranged in a prismatic geometry. In contrast, on the angled nanowires different facets will receive different amount of material flux, and quantum wells with different thicknesses will be formed. Schematic of the growth process with the corresponding structures is shown in figure 12(a), while SEM image of overgrown nanowires is shown in figure 12(b). As can be seen on the inset the droplet is completely crystallized due to the high As flux required for the growth of the $\mathrm{p}-\mathrm{QWs}$.

\subsection{Structural characterization}

With a purpose of detailed investigations of the quality of the growth on the side facets of the nanowires we performed high-resolution TEM characterization on the cross section of the obtained heterostructures. Here, it should be noted that for TEM characterization instead of AlGaAs, pure AlAs was deposited providing better contrast for the measurements. These measurements require a special sample preparation as explained in the following: the nanowires were removed mechanically from the growth substrate as all ready described above, and transferred on Si pieces, with a special care of obtaining high-density parallel oriented nanowires. Afterward they were embedded in $100 \mathrm{~nm}$ thick $\mathrm{SiO}_{2}$ deposited by a sputtering method. In order to obtain the required cross section thin slices of the sample were prepared by means of traditional thin film cross section preparation with the use of a focused ion beam machine. Cross section TEM analysis was performed on both vertically and angled grown nanowires in order to prove that two different kinds of heterostructures will be formed. Furthermore, with a purpose to define a relation between the nominal thickness of material and the material that actually deposited on the side facets, measurements were performed on samples where layers with different thicknesses were grown. 


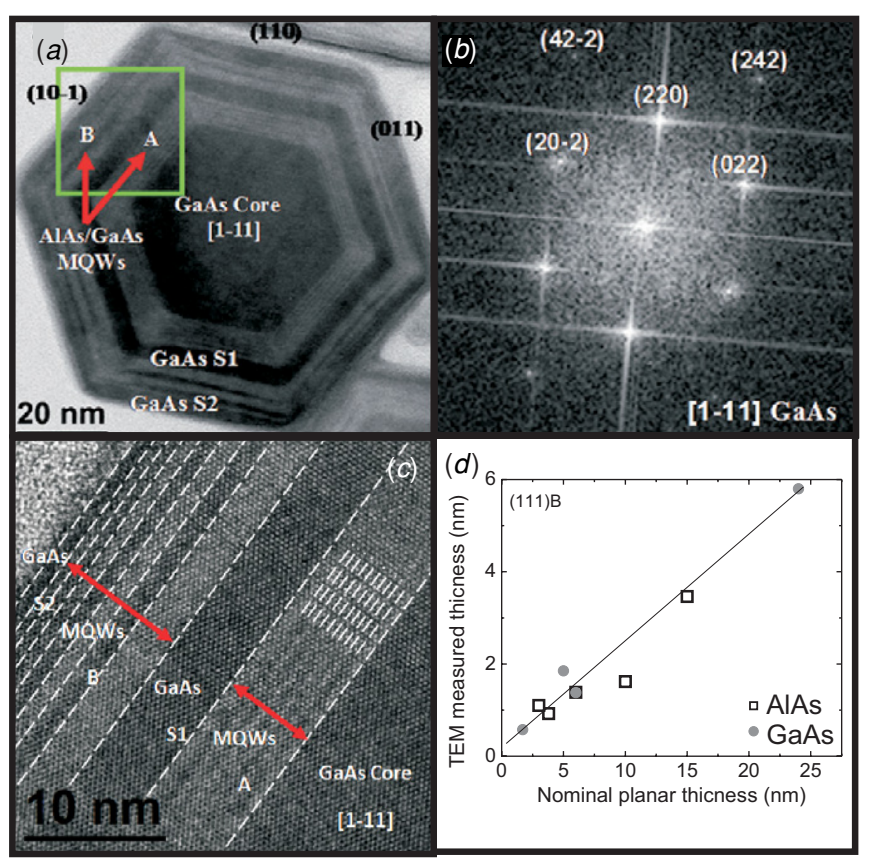

Figure 13. (a) Cross sectional TEM of single prismatic quantum heterostructure. The bright stripes are the AlAs layers, while the dark stripes correspond to GaAs layers. (b) Power spectrum of the presented TEM. The nanowire grows in the $(1-11)$ direction, while the lateral faceting belongs to the $\left\{\begin{array}{lll}1 & 1 & 0\end{array}\right\}$ family. $(c)$ High-resolution TEM from the selected region in $(a)$. A perfect epitaxy between the layers is observable. $(d)$ Correlation between the TEM measured thickness and the nominal deposited thickness both for GaAs and AlAs. The both dependences follow the same linear curve.

3.2.1. Growth on vertical nanowires. On the side facets of the nanowires we have deposited several layers of GaAs and AlAs with different thicknesses. First, we started with deposition of alternating GaAs and AlAs with nominal thickness of few nanometers. Each layer was repeated 6 times, and this region of the sample is labeled as MQWs A-MQWs referring to multiquantum wells. Afterward a thicker layer of GaAs was deposited followed again by alternating layers of GaAs and AlAs with thicknesses ranging from $3 \mathrm{~nm}$ up to $24 \mathrm{~nm}$. This part of the heterostructure is labeled as MQWs B. The growth was finished by deposition of a thin layer of GaAs with a purpose to protect the lower laying AlAs layers from oxidation.

A typical cross sectional TEM measurement of one of the prismatic quantum heterostructures is shown in figure 13(a). The bright stripes correspond to the AlAs layers, while the dark stripes correspond to the GaAs layers. As was expected from the above discussion, due to the directionality of the molecular beams and the rotation of the substrate in the MBE chamber during the growth each facet received the same amount of material which resulted in equal thickness of the layers on all facets. The nanowire conserved the hexagonal shape after the growth which verifies the epitaxial growth mode. In figure $13(b)$, the power spectrum is shown. As can be seen the nanowire grows in the $(1-11)$ while the faceting lateral planes belong to the $\left\{\begin{array}{lll}1 & 1 & 0\end{array}\right\}$ family. A high-resolution TEM from the selected region in figure 13(a) is shown in figure 13(c). Here, in the regions labeled as MQWs A and MQWs B we can clearly observe the perfect epitaxy of the different layers. With the help of the high-resolution TEM analysis we have measured the thickness of each deposited layer and we have compared it with the nominal thickness of deposited material as calibrated on a planar substrate. The results are shown in figure $13(d)$ where the TEM measured thickness versus the nominal deposited thickness is plotted, both for GaAs and AlAs. As can be seen, both dependences follow the same linear curve, which shows that the growth on the side facets is a result of a direct deposition, while the diffusion on the substrate plays a negligible role.

\subsubsection{Growth on angled wires. A series of different} thicknesses of GaAs and AlAs layers, ranging from $8 \mathrm{~nm}$ to $30 \mathrm{~nm}$, was also grown on the side facets of angled nanowires. As schematized in figure 12(a) (lower panel) due to the directionality of the molecular beam the top facets should receive most of the flux, resulting in thickest layers. In contrast, the growth on the side facets should result in lower thickness, while on the facets facing the substrate almost no growth should take place. This is verified by the cross sectional TEM of one of these structures shown in figure 14(a). Again, the bright stripes correspond to AlAs layers, while the dark stripes correspond to GaAs layers.

We have correlated again the TEM measured thickness of the layers with the nominal deposited thickness. The resulting data are plotted in figure 14(b) for the top facet as well as for the side facets, both for GaAs and AlAs. As can be seen the different materials follow the same dependence. As discussed above, this proves that the growth is a result of atoms impinging directly on the surface of the nanowires, while the diffusion on the substrate plays a minor role.

\subsection{Optical properties of $p-Q W s$}

The optical properties of the prismatic heterostructures were investigated with the same confocal microscope setup, described above. With a purpose to have functional prismatic heterostructures it is important that the thickness of the grown layers is uniform along the length of the nanowire. Therefore, we have performed growths on several temperatures in the range from $650{ }^{\circ} \mathrm{C}$ to $465{ }^{\circ} \mathrm{C}$ for finding the optimal growth conditions. In contrast to the samples used for TEM measurements, here we have grown $\mathrm{Al}_{0.35} \mathrm{Ga}_{0.65} \mathrm{As}$. The photoluminescence was recorded at $4.2 \mathrm{~K}$ and sample preparation is already described previously.

Initially, the growth was performed on a substrate temperature of $650{ }^{\circ} \mathrm{C}$. The objective was to avoid deposition of material on the $\mathrm{SiO}_{2}$ surface. PL measurements showed that very small number of nanowires present emission at energies above the band gap of GaAs $(1.51 \mathrm{eV})$ that should result from carrier confinement in the formed quantum wells on the side facets. One representative measurement is shown in figure $15(a)$ where the PL emission is plotted for several excitation powers. As can be seen the emission is centered around $1.60 \mathrm{eV}$, but is much weaker than the emission of the core (centered at $1.51 \mathrm{eV}$, as discussed above) which is anticipated by its tail seen on the lower energy side of the spectrum. 


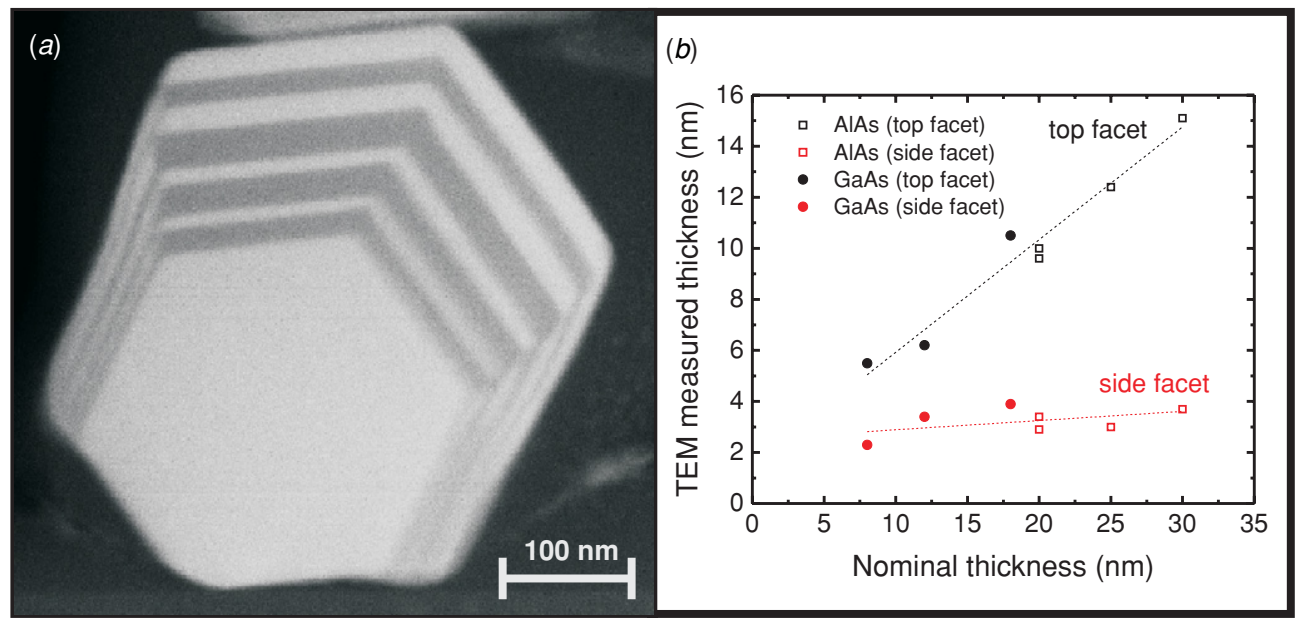

Figure 14. (a) Cross sectional TEM from a single heterostructure. Due to the directionality of the molecular beam the thickness of the grown layers is different for different side facets. (b) Correlation between the TEM measured thickness of the layers and the nominally deposited thickness for both GaAs and AlAs for the top and side facet of the nanowire. The different layers follow the same dependence.
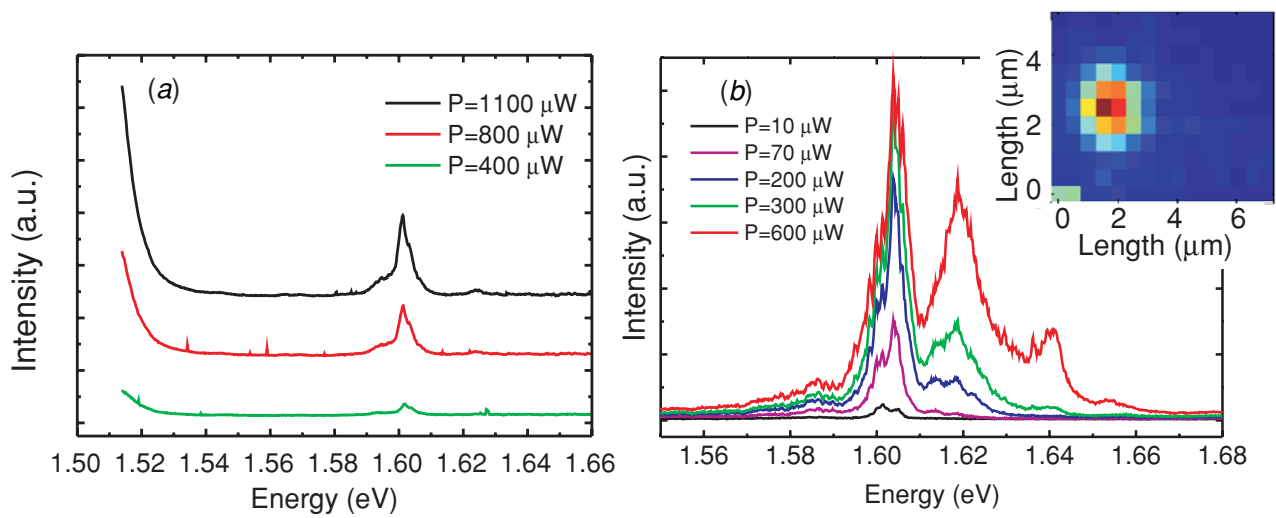

Figure 15. (a) PL spectrum from a p-QW grown on $650{ }^{\circ} \mathrm{C}$. The PL emission is localized at around $1.60 \mathrm{eV}$, and is much weaker than the emission of the core, anticipated by its tale. (b) PL spectrum from a p-QW grown on $600{ }^{\circ} \mathrm{C}$. The emission is characterized with several broad peaks pointing to a non-uniform growth. In the inset, a spatially resolved PL is shown. The p-QW emission is present only at one small part of the nanowire.

Subsequently the temperature was lowered to $600{ }^{\circ} \mathrm{C}$. A PL spectrum of a p-QWs grown on $600{ }^{\circ} \mathrm{C}$ recorded at different excitation powers is shown in figure $15(b)$. Again, we have observed that these emissions are much weaker than the emission of the core of the nanowire (not shown here). The spectrum is characterized with one or more sharp lines at low power, which eventually evolve in broad emission peak at high excitation power. This shows that the formed QW has nonuniform thickness along the nanowire resulting in different energy levels of the localized electrons and holes. In the inset of the figure, a spatially resolved PL is shown. We can see that the emission originating from the $\mathrm{p}-\mathrm{QW}$ is present only on one small part of the nanowire. These findings point out that for these growth conditions, the layer thickness as well as the material quality are non-uniform along the nanowire.

PL measurements performed on growth temperature of $550{ }^{\circ} \mathrm{C}$ also showed that this temperature as well, is not suitable for high quality growth of the p-QWs. The best PL characteristics we obtained from growth realized at $465^{\circ} \mathrm{C}$. A spatially resolved PL measurement from a single nanowire is shown in figure $16(a)$. The emission of the $\mathrm{p}-\mathrm{QWs}$ is characterized with a single narrow line centered at $1.55 \mathrm{eV}$. More important, this emission is much stronger than the emission of the core of the nanowire and extends along its entire length indicating a formation of a $\mathrm{p}-\mathrm{QW}$ with a homogeneous thickness. This proves that the best conformity of the $\mathrm{p}-\mathrm{QWs}$ is obtained at this growth temperature. A spectrum recorded at one point of the nanowires is plotted in figure $16(b)$. The FWHM is $5 \mathrm{meV}$ and lies in the range of results obtained for QWs grown on planar (1 110$)$ GaAs substrates $[51,52]$. This acknowledges the fact that very high quality growth with MBE can be achieved as well on nonplanar substrates.

By applying these optimal growth conditions, we have changed the nominal deposited thickness for the formation of the quantum well. The corresponding PL spectra are plotted in figure 17(a). Indeed, with a decrease of the deposited thickness the emission energy shifts toward larger energies, due to the higher confinement in thinner quantum wells. It should be noted that these emission energies correspond to emissions from thinner quantum wells than the actual deposited values. As was discussed above, and confirmed with 

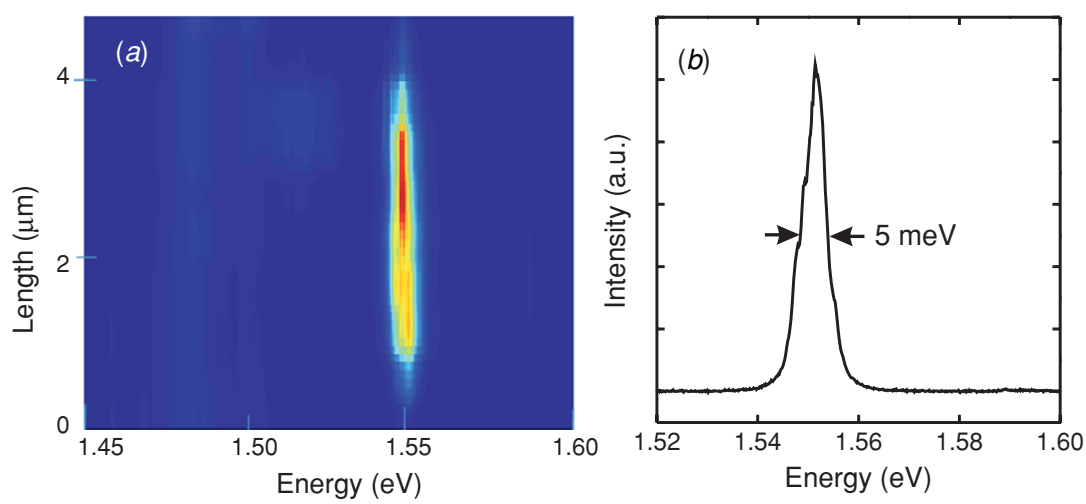

Figure 16. (a) Spatially resolved PL spectroscopy of a single p-QWs heterostructure. The emission from the formed p-QWs is characterized with a single emission line extending along the entire length of the nanowire. $(b)$ PL spectra recorded at one point of the nanowire. The FWHM is $5 \mathrm{meV}$, comparable to results obtained for planar quantum wells grown on (110) GaAs substrates [51, 52].
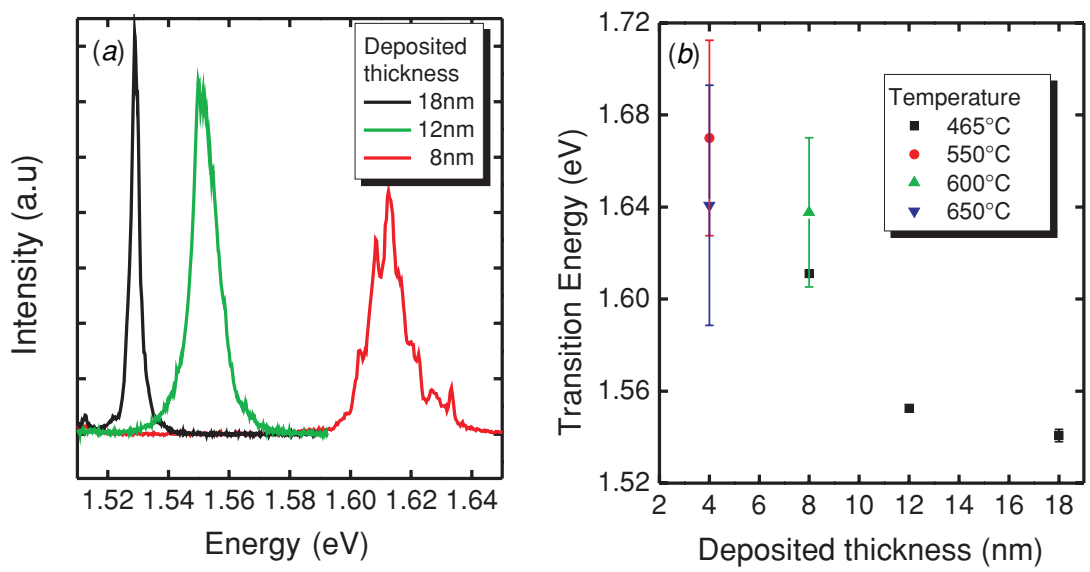

Figure 17. (a) PL spectra from p-QWs with different thicknesses grown on temperature of $465{ }^{\circ} \mathrm{C}$. As the thickness is decreased, the emission energy shifts to higher energies due to the larger confinement for electrons and holes in thinner quantum wells. The sharp peaks in the spectra from the thinner quantum well originate from confinement of carriers in fluctuation of the quantum well width. $(b)$ Transition energies of $\mathrm{p}$-QWs with different thicknesses grown on different temperatures. The standard deviation is dramatically decreased when the temperature is lowered to $465^{\circ} \mathrm{C}$.

the high-resolution TEM measurements, due to the geometry of the nanowires, the grown thickness of materials is smaller than the actual deposited thickness. Nevertheless, we show that we are able to control the emission energy of the p-QWs by changing their thickness. Another point to be noted is the change of the FWHM with respect to the change in the thickness of the quantum well. As the well is thicker, the emission line width is narrower. In the case of thin quantum well (red spectra in figure $17(a)$ ) many sharp peaks are also observable. They are observable at low excitation powers and originate from local confinement of electrons and holes due to small fluctuations in the quantum well width.

At last, we have compared the optical properties of the p-QWs with different thicknesses grown on different growth temperatures. In figure $17(b)$, the dependence of the transition energy from the deposited thickness for different temperatures is plotted. Here, transition energy refers to the energy position of the p-QWs emission. It should also be noted that with a purpose to estimate the standard deviation of the transition energies, several nanowires for each temperature were probed. For high-temperature growth the standard deviation of the transition energy is significantly increased. As has been shown, these were far from the optimal growth conditions. At temperature of $465{ }^{\circ} \mathrm{C}$ the standard deviation decreases dramatically, in agreement with a peak emission with a small FWHM and a small dispersion between nanowires. This confirms again that this temperature results in the best quality of the p-QWs.

\section{Outlook}

GaAs nanowires were grown by using the Ga-assisted growth method with molecular beam epitaxy. We have found out that it is actually the As who is governing the growth and the role of the $\mathrm{Ga}$ is to act as a reservoir for collecting the As atoms. The grown nanowires possess excellent quality as proved by photoluminescence measurements. By using the versatility of growth modes offered by the MBE technique we have add additional functionality to the nanowires by forming the $\mathrm{p}$ QWs. We have shown that their geometry can be changed with varying the orientation of the nanowires with respect to the GaAs substrate. The epitaxy on the side facets of the 
(a)

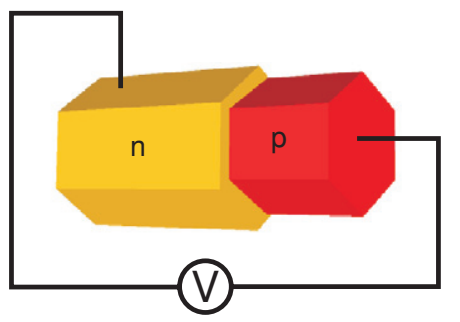

(b)

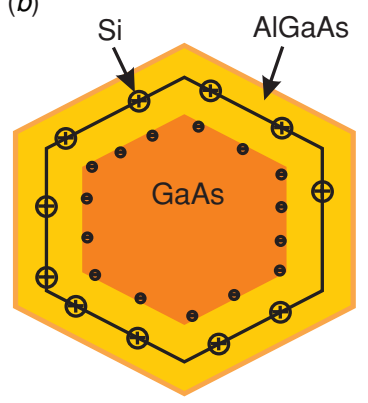

Figure 18. (a) Schematical representation of radial $\mathrm{p}-\mathrm{n}$ junction formed in a nanowire. Both the $\mathrm{p}$ and the $\mathrm{n}$ doping can be achieved by using the amphoteric nature of $\mathrm{Si}$ in GaAs. (b) Schematic representation of a cross section of prismatic heterostructures with a formed two-dimensional electron gas at the GaAs/AlGaAs interface.

nanowires is extremely precise, as proved with high-resolution TEM measurements. Furthermore, these p-QWs presented excellent optical quality and we are able to tune their emission energy by changing their thickness in a controlled way.

This growth technique offers possibilities for in situ doping of the nanowires. It has been shown recently that doped GaAs nanowires can be used as polarizationdependent photodetectors [53]. When the doping is applied to the prismatic heterostructures a variety of functional nanostructures can be obtained. The simplest radial or prismatic structures that one can think of are radial $\mathrm{p}-\mathrm{n}$ or $\mathrm{p}-\mathrm{i}-\mathrm{n}$ junctions as shown schematically in figure 18(a). Such structures are promising candidates for an enhanced solar energy collection. Indeed, the advantage of these radial devices consists in the fact that the generated electron-hole pairs can be separated in a very short distance, thereby reducing the recombination probability [54-56]. A much more refined structure is the fabrication of a two-dimensional electron gas arranged in a prismatic geometry around the nanowire [57, 58]. This can be achieved when during the overgrowth of the side facets of the nanowires with AlGaAs a Si layer is added. Si atoms will act as donors giving electrons. As in the conventional planar modulation doped heterostructures, the electrons will accumulate at the interface of the nanowire core and the AlGaAs grown on the side facets. Thus, prismatically oriented two-dimensional electron gas will be formed, as schematically shown in figure $18(b)$. For sure this will lead toward novel application of these systems especially in the area of high-mobility devices based on GaAs nanowires.

\section{Acknowledgments}

This review is the result of team work. We thank $\mathrm{C}$ Colombo, M Hei $\beta$ and M Heigoldt for the nice experience of working together on this project. We thank M Bichler for his technical help with the MBE. We also thank our collaborators from Universitat de Barcelona Dr J Arbiol and Dr J R Morante for the TEM analysis. Fundings through Marie Curie Excellence Grant SENFED, the DFG program Nanosystems Initiative Munich and SFB 631 are kindly acknowledged. Equally

important, we greatly thank the presidential fellowship of the TU Munich for financing the first two years of this doctoral thesis.

\section{References}

[1] Hiruma K, Yazawa M, Katsuyama T, Ogawa K, Haraguchi K, Koguchi M and Kakibayashi H 1995 J. Appl. Phys. 77447

[2] Schmidt V, Riel H, Senz S, Karg S, Riess W and Gösele U 2006 Samll 285

[3] Minot D E, Kelkensberg F, van Kouwen M, van Dam J A, Kouwenhoven L P, Zwiller V, Borgstrom M T, Wunnicke O, Verheijen M A and Bakkers E P A M 2007 Nano Lett. 7367

[4] De Franceschi S, van Dam J A, Bakkers E P A M, Feiner L F, Gurevich L and Kouwenhoven L P 2003 Appl. Phys. Lett. 83344

[5] Lu W, Xiang J, Timko B P, Wu Y and Lieber C M 2005 Proc. Natl Acad. Sci. 10210046

[6] Duan X, Huang Y, Cui Y, Wang J and Lieber CM 2005 Nature 40966

[7] Duan X, Huang Y, Agarwal R and Lieber C M 2003 Nature 421241

[8] Björk M T, Ohlsson B J, Sass T, Persson i A, Thelander C, Magnusson M H, Deppert K, Wallenberg L R and Samuelson L 2002 Appl. Phys. Lett. 801058

[9] Fontcuberta i Morral A, Zahler J M, Ahrenkiel S P, Wanlass W and Atwater H A 2003 Appl. Phys. Lett. 835413

[10] Luryi S and Suhir E 1986 Appl. Phys. Lett. 49140

[11] Zubia D and Hersee S D 1999 J. Appl. Phys. 856491

[12] Van Mieghem P, Jain S C, Nijs J and Van Overstraeten R 1994 J. Appl. Phys. 75666

[13] Glas F 2006 Phys. Rev. B 74121302

[14] Wagner R S and Ellis W C 1964 Appl. Phys. Lett. 489

[15] Brotherson S D and Lowther J E 1980 Phys. Rev. Lett. 44606

[16] Wang Y, Schmidt S, Senz S and Gösele U 2006 Nature Nanotechnol. 1186

[17] Zardo I et al 2009 Nanotechnology 20155602

[18] Xiang Y, Cao L, Conesa-Boj S, Arbiol J, Peiro F, Heiß M, Zardo I, Morante J R, Brongersma M and Fontcuberta i Morral A 2009 Nanotechnology 20245608

[19] Xiang Y, Cao L, Arbiol J, Brongersma M and Fontcuberta i Morral A 2009 Appl. Phys. Lett. 94163101

[20] Mandl B, Stangl J, Martensson T, Mikkelsen A, Eriksson J, Karlsson L S, Bauer G, Samuelson L and Seifert W 2006 Nano Lett. 61817

[21] Mattila M, Hakkarainen T, Lipsanen H, Jiang H and Kauppinen I E 2006 Appl. Phys. Lett. 8906311

[22] Noborisaka J, Motohisa J, hara S and Fukui T 2005 Appl. Phys. Lett. 87093109

[23] Poole P J, Lefebvre J and Fraser J 2003 Appl. Phys. Lett. 832055

[24] Brunner K, Abstreiter G, Bohm G, Trankle G and Weimann G 1994 Phys. Rev. Lett. 731138

[25] Fischer F S, Apetrii G, Kunze U, Schuh D and Abstreiter G 2006 Nature Phys. 291

[26] Egeler T, Abstreiter G, Weimann G, Demmel T, Heitmann D, Grambow P and Schlapp W 1990 Phys. Rev. Lett. 651804

[27] Lauhon L, Gudiksen M, Wang D and Lieber C M 2002 Nature 42057

[28] Ledentsov N N 1999 Growth Processes and Surface Phase Equilibria in Molecular Beam Epitaxy (Berlin: Springer)

[29] Chatillon C and Chatain D 1995 J. Cryst. Growth 15191

[30] Mano T and Koguchi N 2005 J. Cryst. Growth 278108

[31] Mano T, Watanabe K, Tsukamoto S, Fujioka H, Oshima M and Koguchi N 2000 J. Cryst. Growth 209504

[32] Watanabe K, Koguchi N and Gotoh Y 2000 Japan. J. Appl. Phys. 39 L79 
[33] Mano T et al 2005 Nano Lett. 5425

[34] Heiss M, Riedelberger E, Spirkoska D, Bichler M, Abstreiter $\mathrm{G}$ and Fontcuberta i Morral A 2008 J. Cryst. Growth 3101049

[35] Fontcuberta i Morral A, Colombo C, Arbiol J, Morante J R and Abstreiter G 2008 Appl. Phys. Lett. 92063112

[36] Colombo C, Spirkoska D, Frimmer M, Abstreiter G and Fontcuberta i Morral A 2008 Phys. Rev. B 77155326

[37] Williams D B and Carter C B 1996 Transmission Electron Microscopy (New York: Plenum)

[38] Bao J, Bell D C, Capasso F, Wagner J, Martensson T, Tragardh J and Samuelson L 2008 Nano Lett. 8836

[39] Spirkoska D, Abstreiter G and Fontcuberta i Morral A 2008 Nanotechnology 19435704

[40] Cardona M and Guntherodt G 1982 Light Scattering in Solids (Berlin: Springer)

[41] Steinebach C, Krahne R, Biese G, Schüller C, Heitmann D and Eberl K 1996 Phys. Rev. B 54 R14281

[42] Krahne R, Chilla G, Schüller C, Carbone L, Kudera S, Mannarini G, Manna L, Heitmann D and Cingolani R 2006 Nano Lett. 6478

[43] Watt M, Sotomayor Torres M C, Arnot G E H and Beaumont P S 1990 Semicond. Sci. Technol. 5285

[44] Ruppin R and Englman R 1970 Rep. Prog. Phys. 33149

[45] Gupta R, Xiong Q, Mahan D G and Eklund P C 2003 Nano Lett. 31745

[46] Adu K W, Xiong Q, Gutierrez H R, Chen G and Eklund P C 2006 Appl. Phys. A 85287
[47] Spirkoska D, Colombo C, Heiss M, Abstreiter G and Fontcuberta i Morral A 2008 J. Phys.: Condens. Matter. 20454225

[48] Fontcuberta i Morral A, Spirkoska D, Arbiol J, Heigoldt M, Morante J R and Abstreiter G 2008 Small 4899

[49] Heigoldt M, Arbiol J, Spirkoska D, Rebled J M, Conesa-Boj S, Abstreiter G, Peiro F, Morante J R and Fontcuberta i Morral A 2009 J. Mater. Chem. 19840

[50] Tejedor P, Smilauer P, Roberts C and Joyce B A 1999 Phys. Rev. B 592341

[51] Yoshita M, Kondo N, Sakaki H, Baba M and Akiyama H 2001 Phys. Rev. B 63075305

[52] Gislason H, Sorensen C B and Hvam J M 1996 Appl. Phys. Lett. 69800

[53] Thunich S, Prechtel L, Spirkoska D, Abstreiter G, Fontcuberta i Morral A and Holleitner A W 2009 Appl. Phys. Lett. 95083111

[54] Kayes B M, Filler M A, Putnam M C, Kelzenberg M D, Lewis N S and Atwater H A 2007 Appl. Phys. Lett. 91103110

[55] Kandala A, Betti T and Fontcuberta i Morral A 2009 Phys. Stat. Solidi A 206173

[56] Colombo C, Heiss M, Graetzel M and Fontcuberta i Morral A 2009 Appl. Phys. Lett. 94173108

[57] Ferrari G, Bertoni A, Goldoni G and Molinari E 2008 Phys. Rev. B 78115326

[58] Ferrari G, Goldoni G, Bertoni A, Cuoghi G and Molinari E 2009 Nano Lett. 91631 